\title{
CHARACTERIZATIONS OF LIE HIGHER AND LIE TRIPLE DERIVATIONS ON TRIANGULAR ALGEBRAS
}

\author{
JiAnkui Li AND QiHua Shen
}

\begin{abstract}
In this paper, we show that under certain conditions every Lie higher derivation and Lie triple derivation on a triangular algebra are proper, respectively. The main results are then applied to (block) upper triangular matrix algebras and nest algebras.
\end{abstract}

\section{Introduction}

Let $\mathcal{R}$ be a commutative ring with identity. A triangular algebra is an algebra of the form

$$
\left(\begin{array}{cc}
\mathcal{A} & \mathcal{M} \\
& \mathcal{B}
\end{array}\right)
$$

where $\mathcal{A}$ and $\mathcal{B}$ are unital algebras over $\mathcal{R}$ and $\mathcal{M}$ is an $(\mathcal{A}, \mathcal{B})$-bimodule which is faithful as a left $\mathcal{A}$-module as well as a right $\mathcal{B}$-module. Basic examples of triangular algebras are upper triangular matrix algebras and nest algebras. Many authors have made important contributions to the related topics, see $[2,4,5,6,13]$. Cheung in [5] initiated the study of linear mappings of abstract triangular algebras and obtained a number of elegant results. He described automorphisms, derivations, commuting mappings and Lie derivations of triangular algebras in $[4,5,6]$. Benkovič [1] studied biderivations of triangular algebras and showed that under certain conditions every biderivation on a triangular algebra is the sum of an extremal biderivation and an inner biderivation. Zhang and $\mathrm{Yu}[16]$ proved that any Jordan derivation on a triangular algebra is a derivation. Xiao and Wei [14] developed this result to the case of Jordan higher derivations and obtained that every Jordan higher derivation on a triangular algebra is a higher derivation.

Let $\mathcal{A}$ be an algebra over a commutative ring $\mathcal{R}$ and $Z(\mathcal{A})$ be its center. Let $\mathbb{N}$ denote the set of non-negative integers. Further, let $[x, y]=x y-y x$ be the commutator (the Lie product) of the elements $x, y \in \mathcal{A}$. Let $D=\left(L_{i}\right)_{i \in \mathbb{N}}$ be a sequence of linear maps on $\mathcal{A}$ such that $L_{0}=i d_{\mathcal{A}}$. $D$ is called a higher

Received April 8, 2011; Revised July 14, 2011.

2010 Mathematics Subject Classification. Primary 47L35; Secondly 16W25.

Key words and phrases. Lie derivation, Lie higher derivation, Lie triple derivation, triangular algebra. 
derivation if $L_{n}(x y)=\sum_{i+j=n} L_{i}(x) L_{j}(y)$ for each $n \in \mathbb{N}$ and $x, y \in \mathcal{A} ; D$ is called a Lie higher derivation if $L_{n}([x, y])=\sum_{i+j=n}\left[L_{i}(x), L_{j}(y)\right]$ for each $n \in \mathbb{N}$ and $x, y \in \mathcal{A}$. It is clear that all higher derivations are Lie higher derivations and the converse is not true in general. Assume that $D=\left(D_{i}\right)_{i \in \mathbb{N}}$ is a higher derivation on $\mathcal{A}$. For any $n \in \mathbb{N}$, let

$$
L_{n}=D_{n}+h_{n}
$$

where $h_{n}: \mathcal{A} \rightarrow Z(\mathcal{A})$ is a linear map such that $h_{n}([x, y])=0$ for each $x, y \in \mathcal{A}$. It is easily checked that $\left(L_{n}\right)_{n \in \mathbb{N}}$ is a Lie higher derivation, and not a higher derivation if $h_{n} \neq 0$ for some $n$. Lie higher derivations of the above form are called proper. The natural problem that one considers in this context is whether or not every Lie higher derivation is proper. In [10], the author discussed the properties of Lie higher derivations. In [11], Qi and Hou showed that each Lie higher derivation is proper on nest algebras. In Section 3, we show that under certain conditions every Lie higher derivation on a triangular algebra is proper. An immediate application recaptures the main result in [11].

Let $\mathcal{A}$ be an algebra over a commutative $\operatorname{ring} \mathcal{R}$ and $Z(\mathcal{A})$ be its center. A linear map $L$ on $\mathcal{A}$ is called a Lie triple derivation if $L([[x, y], z])=$ $[[L(x), y], z]+[[x, L(y)], z]+[[x, y], L(z)]$ for all $x, y, z \in \mathcal{A}$. Lie triple derivations have received a fair amount of attention in recent years, see for example $[7,12,15]$ and references therein. A central problem is whether a Lie triple derivation can be decomposed into a sum of a derivation and a linear map $h: \mathcal{A} \rightarrow Z(\mathcal{A})$ such that $h([[x, y], z])=0$ for all $x, y, z \in \mathcal{A}$. Lie triple derivations of the above form are called proper. It was shown in [9] that each Lie triple derivation on a von Neumann algebra which has no commutative direct summand into itself is proper. Results related to Lie triple derivations on prime algebras were considered in [3]. Lu [7] considered similar questions on nest algebras. In Section 4, we characterize Lie triple derivations on triangular algebras and give necessary and sufficient conditions such that every Lie triple derivation is proper. We then apply the main results to (block) triangular matrix algebras and (continuous) nest algebras.

\section{Triangular algebras}

Let $\mathcal{A}$ and $\mathcal{B}$ be unital algebras over a commutative ring $\mathcal{R}$, and let $\mathcal{M}$ be a unital $(\mathcal{A}, \mathcal{B})$-bimodule, which is faithful as a left $\mathcal{A}$-module and also a right $\mathcal{B}$-module. Recall that a left (respectively, right) $\mathcal{A}$-module $\mathcal{M}$ is faithful if $a \mathcal{M}=0$ (respectively, $\mathcal{M} a=0$ ) implies that $a=0$ for every $a \in \mathcal{A}$. The $\mathcal{R}$-algebra

$$
\mathfrak{A}=\operatorname{Tri}(\mathcal{A}, \mathcal{M}, \mathcal{B})=\left\{\left(\begin{array}{cc}
a & m \\
& b
\end{array}\right): a \in \mathcal{A}, m \in \mathcal{M}, b \in \mathcal{B}\right\}
$$


under the usual matrix operations is called a triangular algebra. We define two natural projections $\pi_{\mathcal{A}}: \mathfrak{A} \rightarrow \mathcal{A}$ and $\pi_{\mathcal{B}}: \mathfrak{A} \rightarrow \mathcal{B}$ by

$$
\pi_{\mathcal{A}}\left(\begin{array}{cc}
a & m \\
& b
\end{array}\right)=a \text { and } \pi_{\mathcal{B}}\left(\begin{array}{cc}
a & m \\
& b
\end{array}\right)=b .
$$

By [6, Proposition 3] we know that the center $Z(\mathfrak{A})$ of $\mathfrak{A}$ coincides with

$$
\left\{\left(\begin{array}{cc}
a & 0 \\
& b
\end{array}\right): a m=m b \text { for all } m \in \mathcal{M}\right\} \text {. }
$$

Moreover, $\pi_{\mathcal{A}}(Z(\mathfrak{A})) \subseteq Z(\mathcal{A})$ and $\pi_{\mathcal{B}}(Z(\mathfrak{A})) \subseteq Z(\mathcal{B})$, and there exists a unique algebra isomorphism $\tau: \pi_{\mathcal{A}}(Z(\mathfrak{A})) \rightarrow \pi_{\mathcal{B}}(Z(\mathfrak{A}))$ such that $a m=m \tau(a)$ for all $m \in \mathcal{M}$.

Let $1_{\mathcal{A}}$ and $1_{\mathcal{B}}$ be identities of the algebras $\mathcal{A}$ and $\mathcal{B}$, respectively, and let 1 be the identity of the triangular algebra $\mathfrak{A}$. Throughout this paper we shall use the following notation

$$
e=\left(\begin{array}{cc}
1_{\mathcal{A}} & 0 \\
& 0
\end{array}\right), f=1-e=\left(\begin{array}{cc}
0 & 0 \\
& 1_{\mathcal{B}}
\end{array}\right) .
$$

Then $e$ and $f$ are orthogonal idempotents of $\mathfrak{A}$ and so $\mathfrak{A}$ may be represented as

$$
\mathfrak{A}=1 \mathfrak{A} 1=(e+f) \mathfrak{A}(e+f)=e \mathfrak{A} e+e \mathfrak{A} f+f \mathfrak{A} f,
$$

where $e \mathfrak{A} e$ is a subalgebra of $\mathfrak{A}$ isomorphic to $\mathcal{A}, f \mathfrak{A} f$ is a subalgebra of $\mathfrak{A}$ isomorphic to $\mathcal{B}$ and $e \mathfrak{A} f$ is a $(e \mathfrak{A} e, f \mathfrak{A} f)$-bimodule isomorphic to the bimodule $\mathcal{M}$. To simplify the notation we will use the following convention: $a=e a e \in$ $\mathcal{A}=e \mathfrak{A} e, b=f b f \in \mathcal{B}=f \mathfrak{A} f$ and $m=e m f \in \mathcal{M}=e \mathfrak{A} f$. Then each element $x=e x e+e x f+f x f \in \mathfrak{A}$ can be represented in the form

$$
x=e x e+e x f+f x f=a+m+b,
$$

where $a \in \mathcal{A}, b \in \mathcal{B}$ and $m \in \mathcal{M}$.

\section{Characterizations of Lie higher derivations}

In this section, we characterize Lie higher derivations on triangular algebras. The main result is stated as follows.

Theorem 3.1. Let $\mathfrak{A}=\operatorname{Tri}(\mathcal{A}, \mathcal{M}, \mathcal{B})$ be a triangular algebra such that $Z(\mathcal{A})=$ $\pi_{\mathcal{A}}(Z(\mathfrak{A}))$ and $Z(\mathcal{B})=\pi_{\mathcal{B}}(Z(\mathfrak{A}))$. Then every Lie higher derivation on $\mathfrak{A}$ is proper. That is to say, $D=\left(L_{n}\right)_{n \in \mathbb{N}}$ is a Lie higher derivation on $\mathfrak{A}$ if and only if $L_{n}$ has the form

$$
L_{n}(x)=\delta_{n}(x)+h_{n}(x), x \in \mathfrak{A},
$$

where $\left(\delta_{n}\right)_{n \in \mathbb{N}}$ is a higher derivation and $h_{n}: \mathfrak{A} \rightarrow Z(\mathfrak{A})$ is a sequence of linear mappings satisfying

$$
h_{n}([x, y])=0, x, y \in \mathfrak{A}, n \in \mathbb{N} .
$$


Remark 3.2. By the definition of Lie higher derivation, it is clear that $L_{1}$ is in fact a Lie derivation. Hence we have by [5, Theorem 3.3.3] that there exist a derivation $\delta_{1}$ on $\mathfrak{A}$ and a linear mapping $h_{1}: \mathfrak{A} \rightarrow Z(\mathfrak{A})$ with

$$
h_{1}([x, y])=0 x, y \in \mathfrak{A}
$$

such that

$$
L_{1}(x)=\delta_{1}(x)+h_{1}(x) x \in \mathfrak{A} .
$$

Moreover, $L_{1}$ and $\delta_{1}$ have the following properties:

$$
L_{1}(e) \in \mathcal{M}+Z(\mathfrak{A}) ; L_{1}(\mathcal{M}) \subseteq \mathcal{M} ;
$$

$\mathbf{P}_{1}$

$$
\begin{aligned}
L_{1}(\mathcal{A}) & \subseteq \mathcal{A}+\mathcal{M}+Z(\mathfrak{A}) ; L_{1}(\mathcal{B}) \subseteq \mathcal{B}+\mathcal{M}+Z(\mathfrak{A}) ; \\
\delta_{1}(e) & \in \mathcal{M} ; \delta_{1}(\mathcal{A}) \subseteq \mathcal{A}+\mathcal{M} ; \delta_{1}(\mathcal{B}) \subseteq \mathcal{B}+\mathcal{M} .
\end{aligned}
$$

Proof of Theorem 3.1. We only need to check the "only if" part. Assume that

$$
D=\left(L_{n}\right)_{n \in \mathbb{N}}
$$

is a Lie higher derivation of $\mathfrak{A}$. We proceed by induction on $n \in \mathbb{N}$.

When $n=1$, the conclusion is true by Remark 3.2. Now we assume that

$$
L_{m}(x)=\delta_{m}(x)+h_{m}(x)
$$

holds for all $x \in \mathfrak{A}$ and for all $m<n \in \mathbb{N}$, where $h_{m}: \mathfrak{A} \rightarrow Z(\mathfrak{A})$ is such that

$$
h_{m}([x, y])=0 \text { for all } x, y \in \mathfrak{A},
$$

and

$$
\delta_{m}(x y)=\sum_{i+j=m} \delta_{i}(x) \delta_{j}(y) \text { for all } x, y \in \mathfrak{A} .
$$

Moreover, $L_{m}$ and $\delta_{m}$ have the following properties:

$$
L_{m}(e) \in \mathcal{M}+Z(\mathfrak{A}) ; L_{m}(\mathcal{M}) \subseteq \mathcal{M} ;
$$

$\mathbf{P}_{\mathbf{m}}$

$$
\begin{aligned}
L_{m}(\mathcal{A}) & \subseteq \mathcal{A}+\mathcal{M}+Z(\mathfrak{A}) ; L_{m}(\mathcal{B}) \subseteq \mathcal{B}+\mathcal{M}+Z(\mathfrak{A}) ; \\
\delta_{m}(e) & \in \mathcal{M} ; \delta_{m}(\mathcal{A}) \subseteq \mathcal{A}+\mathcal{M} ; \delta_{m}(\mathcal{B}) \subseteq \mathcal{B}+\mathcal{M} .
\end{aligned}
$$

Our aim is to show that $L_{n}$ also satisfies the similar properties.

Claim 1. $L_{n}(\mathcal{M}) \subseteq \mathcal{M}$ and $L_{n}(e) \in \mathcal{M}+Z(\mathfrak{A})$.

For any $m \in \mathcal{M}$, let

$$
L_{n}(e)=a_{1}+m_{1}+b_{1}, L_{n}(m)=a_{2}+m_{2}+b_{2} .
$$

Then by $\mathbf{P}_{\mathbf{m}}$, we have

$$
\begin{aligned}
L_{n}(m) & =L_{n}([e, m]) \\
& =\sum_{i+j=n}\left[L_{i}(e), L_{j}(m)\right] \\
& =\left[L_{n}(e), m\right]+\left[e, L_{n}(m)\right] \\
& =a_{1} m-m b_{1}+m_{2} \in \mathcal{M},
\end{aligned}
$$


and so

$$
a_{2}=b_{2}=0 \text {. }
$$

This implies that $L_{n}(m) \in \mathcal{M}$. Hence

$$
L_{n}(m)=m_{2}=a_{1} m-m b_{1}+m_{2},
$$

which implies $a_{1} m=m b_{1}$.

Therefore, $a_{1}+b_{1} \in Z(\mathfrak{A})$ and $L_{n}(e)=a_{1}+m_{1}+b_{1} \in \mathcal{M}+Z(\mathfrak{A})$.

Claim 2. $L_{n}(\mathcal{A}) \subseteq \mathcal{A}+\mathcal{M}+Z(\mathfrak{A}) ; L_{n}(\mathcal{B}) \subseteq \mathcal{B}+\mathcal{M}+Z(\mathfrak{A})$.

For any $a \in \mathcal{A}$ and $b \in \mathcal{B}$, let

$$
L_{n}(a)=a_{1}+m_{1}+b_{1}, L_{n}(b)=a_{2}+m_{2}+b_{2} .
$$

Then

$$
\begin{aligned}
0 & =L_{n}([a, b]) \\
& =\sum_{i+j=n}\left[L_{i}(a), L_{j}(b)\right] \\
& =\left[L_{n}(a), b\right]+\sum_{\substack{i+j=n \\
i \neq 0, n}}\left[L_{i}(a), L_{j}(b)\right]+\left[a, L_{n}(b)\right] \\
& =\left[m_{1}, b\right]+\left[b_{1}, b\right]+\left[a, a_{2}\right]+\left[a, m_{2}\right]+\sum_{\substack{i+j=n \\
i \neq 0, n}}\left[L_{i}(a), L_{j}(b)\right] .
\end{aligned}
$$

Since $L_{i}(a) \in \mathcal{A}+\mathcal{M}+Z(\mathfrak{A})$ and $L_{i}(b) \in \mathcal{B}+\mathcal{M}+Z(\mathfrak{A})$ for $1 \leq i \leq n-1$, we have

Hence

$$
\sum_{\substack{i+j=n \\ i \neq 0, n}}\left[L_{i}(a), L_{j}(b)\right] \in \mathcal{M} .
$$

which implies

$$
\left[b_{1}, b\right]=\left[a, a_{2}\right]=0
$$

Therefore, our assumptions on $\mathfrak{A}$ yield

$$
b_{1} \in Z(\mathcal{B}), a_{2} \in Z(\mathcal{A}) .
$$

$$
\begin{aligned}
L_{n}(a) & =\left(a_{1}-\tau^{-1}\left(b_{1}\right)\right)+m_{1}+\left(\tau^{-1}\left(b_{1}\right)+b_{1}\right) \in \mathcal{A}+\mathcal{M}+Z(\mathfrak{A}), \\
L_{n}(b) & =\left(a_{2}+\tau a_{2}\right)+m_{2}+\left(b_{2}-\tau a_{2}\right) \in \mathcal{B}+\mathcal{M}+Z(\mathfrak{A}) .
\end{aligned}
$$

Now, for any $x=a+m+b$, where $a \in \mathcal{A}, b \in \mathcal{B}$ and $m \in \mathcal{M}$, suppose

$$
L_{n}(a)=a_{1}+m_{1}+b_{1}, L_{n}(b)=a_{2}+m_{2}+b_{2} \text { and } L_{n}(m)=m_{3} \text {. }
$$

Let

and let

$$
\begin{gathered}
h_{n}(a)=\tau^{-1}\left(b_{1}\right)+b_{1}, h_{n}(b)=a_{2}+\tau a_{2}, h_{n}(m)=0, \\
h_{n}(x)=h_{n}(a)+h_{n}(m)+h_{n}(b),
\end{gathered}
$$

$$
\delta_{n}(x)=L_{n}(x)-h_{n}(x) .
$$


Clearly $h_{n}: \mathfrak{A} \rightarrow Z(\mathfrak{A})$. By the linearity of $L_{n}$ and $\tau$, it is easy to verify that $h_{n}$ and $\delta_{n}$ are linear. Also from the definition of $\delta_{n}$, it follows from Claims 1 and 2 that

$$
\delta_{n}(\mathcal{M}) \subseteq \mathcal{M}, \delta_{n}(\mathcal{A}) \subseteq \mathcal{A}+\mathcal{M}, \delta_{n}(\mathcal{B}) \subseteq \mathcal{B}+\mathcal{M}
$$

Claim 3. $\delta_{n}$ has the following properties:

(1) $\delta_{n}(a m)=\sum_{i+j=n} \delta_{i}(a) \delta_{j}(m)$ for all $a \in \mathcal{A}$ and $m \in \mathcal{M}$.

(2) $\delta_{n}(m b)=\sum_{i+j=n} \delta_{i}(m) \delta_{j}(b)$ for all $m \in \mathcal{M}$ and $b \in \mathcal{B}$.

(3) $\delta_{n}(a b)=\sum_{i+j=n} \delta_{i}(a) \delta_{j}(b)$ for all $a \in \mathcal{A}$ and $b \in \mathcal{B}$.

(4) $\delta_{n}\left(a_{1} a_{2}\right)=\sum_{i+j=n} \delta_{i}\left(a_{1}\right) \delta_{j}\left(a_{2}\right)$ for all $a_{1}, a_{2} \in \mathcal{A}$.

(5) $\delta_{n}\left(b_{1} b_{2}\right)=\sum_{i+j=n} \delta_{i}\left(b_{1}\right) \delta_{j}\left(b_{2}\right)$ for all $b_{1}, b_{2} \in \mathcal{B}$.

In fact, for any $a \in \mathcal{A}$ and $m \in \mathcal{M}$, we have

$$
\begin{aligned}
\delta_{n}(a m) & =L_{n}([a, m]) \\
& =\sum_{i+j=n}\left[L_{i}(a), L_{j}(m)\right] \\
& =\sum_{i+j=n}\left[\delta_{i}(a)+h_{i}(a), \delta_{j}(m)+h_{j}(m)\right] \\
& =\sum_{i+j=n}\left[\delta_{i}(a), \delta_{j}(m)\right] .
\end{aligned}
$$

Since

$$
\delta_{i}(a) \in \mathcal{A}+\mathcal{M}, \delta_{i}(m) \in \mathcal{M}, 1 \leq i \leq n
$$

the equation (3.1) becomes

$$
\delta_{n}(a m)=\sum_{i+j=n} \delta_{i}(a) \delta_{j}(m) .
$$

(2) and (3) may go similarly.

For any $a_{1}, a_{2} \in \mathcal{A}$ and $m \in \mathcal{M}$, by (1) we have

$$
\delta_{n}\left(a_{1} a_{2} m\right)=\sum_{i+j=n} \delta_{i}\left(a_{1} a_{2}\right) \delta_{j}(m)=\sum_{\substack{i+j=n \\ j \neq 0}} \delta_{i}\left(a_{1} a_{2}\right) \delta_{j}(m)+\delta_{n}\left(a_{1} a_{2}\right) m
$$

and

$$
\begin{aligned}
\delta_{n}\left(a_{1} a_{2} m\right) & =\sum_{i+j=n} \delta_{i}\left(a_{1}\right) \delta_{j}\left(a_{2} m\right)=\sum_{i+j+k=n} \delta_{i}\left(a_{1}\right) \delta_{j}\left(a_{2}\right) \delta_{k}(m) \\
& =\sum_{\substack{i+j+k=n \\
k \neq 0}} \delta_{i}\left(a_{1}\right) \delta_{j}\left(a_{2}\right) \delta_{k}(m)+\sum_{i+j=n} \delta_{i}\left(a_{1}\right) \delta_{j}\left(a_{2}\right) m .
\end{aligned}
$$

By our assumption on $\delta_{m}(m<n)$, the above equation yields

$$
\delta_{n}\left(a_{1} a_{2}\right) m=\sum_{i+j=n} \delta_{i}\left(a_{1}\right) \delta_{j}\left(a_{2}\right) m
$$


for any $m \in \mathcal{M}$. By faithfulness of $\mathcal{M}$, we obtain

$$
\left.\delta_{n}\left(a_{1} a_{2}\right)\right|_{\mathcal{A}}=\left.\left(\sum_{i+j=n} \delta_{i}\left(a_{1}\right) \delta_{j}\left(a_{2}\right)\right)\right|_{\mathcal{A}} .
$$

Similarly, for any $a_{1}, a_{2} \in \mathcal{A}$ and $b \in \mathcal{B}$, it follows from (3) that

$$
\delta_{n}\left(a_{1} a_{2}\right) b=\sum_{i+j=n} \delta_{i}\left(a_{1}\right) \delta_{j}\left(a_{2}\right) b
$$

taking $b=I_{\mathcal{B}}$ yields

$$
\left.\delta_{n}\left(a_{1} a_{2}\right)\right|_{\mathcal{M}}=\left.\left(\sum_{i+j=n} \delta_{i}\left(a_{1}\right) \delta_{j}\left(a_{2}\right)\right)\right|_{\mathcal{M}}
$$

Now the equations (3.2) and (3.3) give (4) of Claim 3.

Similarly, one can check that (5) of Claim 3 holds for all $b_{1}, b_{2} \in \mathcal{B}$.

Claim 4. $\delta_{n}(x y)=\sum_{i+j=n} \delta_{i}(x) \delta_{j}(y)$ for all $x, y \in \mathfrak{A}$.

For any $x, y \in \mathfrak{A}$,

$$
x=a_{1}+m_{1}+b_{1}, y=a_{2}+m_{2}+b_{2},
$$

where $a_{1}, a_{2} \in \mathcal{A}, m_{1}, m_{2} \in \mathcal{M}$ and $b_{1}, b_{2} \in \mathcal{B}$. By Claim 3, it is easy to check that

$$
\delta_{n}(x y)=\sum_{i+j=n} \delta_{i}(x) \delta_{j}(y)
$$

Claim 5. $h_{n}([x, y])=0$ for all $x, y \in \mathfrak{A}$.

For any $x, y \in \mathfrak{A}$,

$$
\begin{aligned}
h_{n}([x, y])= & L_{n}([x, y])-\delta_{n}([x, y]) \\
= & \sum_{i+j=n}\left[L_{i}(x), L_{j}(y)\right]-\delta_{n}(x y)+\delta_{n}(y x) \\
= & \sum_{i+j=n}\left[\delta_{i}(x)+h_{i}(x), \delta_{j}(y)+h_{j}(y)\right] \\
& -\sum_{i+j=n} \delta_{i}(x) \delta_{j}(y)+\sum_{i+j=n} \delta_{i}(y) \delta_{j}(x) \\
= & \sum_{i+j=n}\left[\delta_{i}(x), \delta_{j}(y)\right]-\sum_{i+j=n} \delta_{i}(x) \delta_{j}(y)+\sum_{i+j=n} \delta_{i}(y) \delta_{j}(x) \\
= & 0 .
\end{aligned}
$$

The proof of the theorem is completed. 
Let $\mathbb{N}$ be the set of all positive integers and let $n \in \mathbb{N}$. For every positive integers $m, m \leq n$, we denote by $\bar{k}=\left(k_{1}, k_{2}, \ldots, k_{m}\right) \in \mathbb{N}^{m}$ an ordered $m$ vector of positive integers such that $n=k_{1}+k_{2}+\cdots+k_{m}$. The block upper triangular matrix algebra $B_{n}^{\bar{k}}(\mathcal{R})$ is a subalgebra of $M_{n}(\mathcal{R})$ of the form

$$
B_{n}^{\bar{k}}(\mathcal{R})=\left(\begin{array}{cccc}
M_{k_{1}}(\mathcal{R}) & M_{k_{1} \times k_{2}}(\mathcal{R}) & \cdots & M_{k_{1} \times k_{m}}(\mathcal{R}) \\
0 & M_{k_{2}}(\mathcal{R}) & \cdots & M_{k_{2} \times k_{m}}(\mathcal{R}) \\
\vdots & \vdots & \ddots & \vdots \\
0 & 0 & \cdots & M_{k_{m}}(\mathcal{R})
\end{array}\right)
$$

Let $M_{k \times m}(\mathcal{R})$ denote the set of all $k \times m$ matrices and $T_{n}(\mathcal{R})$ denote the algebra of all $n \times n$ upper triangular matrices over $\mathcal{R}$, then it is easily seen that $M_{n}(\mathcal{R})$ and $T_{n}(\mathcal{R})$ are two special cases of block upper triangular matrix algebras. If we have $n \geq 2$ and $B_{n}^{\bar{k}}(\mathcal{R}) \neq M_{n}(\mathcal{R})$, then $B_{n}^{\bar{k}}(\mathcal{R})$ is a triangular algebra and can be represented as

$$
B_{n}^{\bar{k}}(\mathcal{R})=\left(\begin{array}{cc}
B_{l}^{\overline{k_{1}}}(\mathcal{R}) & M_{l \times(n-l)}(\mathcal{R}) \\
& B_{n-l}^{k_{2}}(\mathcal{R})
\end{array}\right)=\left(\begin{array}{cc}
\mathcal{A} & \mathcal{M} \\
& \mathcal{B}
\end{array}\right),
$$

where $l \in\left\{k_{1}, k_{1}+k_{2}, \ldots, k_{1}+k_{2}+\cdots+k_{m-1}\right\}$ and $\overline{k_{1}} \in \mathbb{N}^{l}, \overline{k_{2}} \in \mathbb{N}^{m-l}$. Since $Z\left(B_{n}^{\bar{k}}\right)=\mathcal{R} 1, Z(\mathcal{A})=\mathcal{R} 1_{\mathcal{A}}$ and $Z(\mathcal{B})=\mathcal{R} 1_{\mathcal{B}}$, as an corollary to our main theorem, we have:

Corollary 3.3. Let $\mathcal{R}$ be a commutative ring with identity. Every Lie higher derivation on $B_{n}^{\bar{k}}(\mathcal{R})\left(\neq M_{n}(\mathcal{R})\right)$ is proper. In particular, every Lie higher derivation on $T_{n}(\mathcal{R})$ is proper.

Let $\mathcal{N}$ be a nest on a Banach space $X$ over the real or complex field $\mathbb{F}$ and $\operatorname{alg} \mathcal{N}$ be the associated algebra. Suppose there exists a non-trivial element $N_{1} \in \mathcal{N}$ which is complemented in $X$, that is, there exists an idempotent $P \in \operatorname{alg} \mathcal{N}$ such that $\operatorname{ran} P=N_{1}$. Let $\mathcal{A}=P \operatorname{alg} \mathcal{N} P, \mathcal{M}=P \operatorname{alg} \mathcal{N}(I-P)$ and $\mathcal{B}=(I-P) \operatorname{alg} \mathcal{N}(I-P)$. Then $\mathcal{M}$ is a faithful $(\mathcal{A}, \mathcal{B})$-bimodule, and alg $\mathcal{N}=\operatorname{Tri}(\mathcal{A}, \mathcal{M}, \mathcal{B})$ is an upper triangular algebra. Note that $Z(\operatorname{alg} \mathcal{N})=\mathbb{F} I$. Thus the following corollary is immediate.

Corollary 3.4 ([11]). Let $\mathcal{N}$ be a nest on a Banach space $X$ over the real or complex field $\mathbb{F}$. If there exists a non-trivial element in $\mathcal{N}$ which is complemented in $X$, then $D=\left(L_{n}\right)_{n \in \mathbb{N}}$ is a Lie higher derivation of the nest algebra alg $\mathcal{N}$ if and only if $L_{n}$ has the form

$$
L_{n}(x)=\delta_{n}(x)+h_{n}(x) I, x \in \mathfrak{A},
$$

where $\left(\delta_{n}\right)_{n \in \mathbb{N}}$ is a higher derivation and $\left(h_{n}\right)_{n \in \mathbb{N}}$ is a sequence of linear functionals satisfying

$$
h_{n}([x, y])=0, x, y \in \mathfrak{A}, n \in \mathbb{N} .
$$




\section{Characterizations of Lie triple derivations}

In this section, we characterize Lie triple derivations on triangular algebras. For a subset $\mathcal{B}$ of an algebra $\mathcal{A}$, we denote $\mathcal{B}^{\prime}$ the commutant of $\mathcal{B}$ in $\mathcal{A}$. In other words,

$$
\mathcal{B}^{\prime}=\{a \in \mathcal{A}: a b=b a \text { for every } b \in \mathcal{B}\} .
$$

Proposition 4.1. A Lie triple derivation on $\mathfrak{A}=\operatorname{Tri}(\mathcal{A}, \mathcal{M}, \mathcal{B})$ is of the form

$$
L\left(\begin{array}{cc}
a & m \\
& b
\end{array}\right)=\left(\begin{array}{cc}
A_{11}(a)+B_{11}(b) & a n_{0}-n_{0} b+C_{12}(m) \\
A_{22}(a)+B_{22}(b)
\end{array}\right),
$$

where $n_{0} \in \mathcal{M}, A_{11}: \mathcal{A} \rightarrow \mathcal{A}, B_{22}: \mathcal{B} \rightarrow \mathcal{B}, A_{22}: \mathcal{A} \rightarrow[\mathcal{B}, \mathcal{B}]^{\prime}, B_{11}: \mathcal{B} \rightarrow$ $[\mathcal{A}, \mathcal{A}]^{\prime}, C_{12}: \mathcal{M} \rightarrow \mathcal{M}$ are linear mappings satisfying

(i) $A_{11}$ is a Lie triple derivation on $\mathcal{A}$, $\left[\left[A_{22}(a), b_{1}\right], b_{2}\right]=0, A_{22}\left(\left[\left[a_{1}, a_{2}\right], a_{3}\right]\right)$ $=0, C_{12}(a m)=A_{11}(a) m-m A_{22}(a)+a C_{12}(m)$;

(ii) $B_{22}$ is a Lie triple derivation on $\mathcal{B},\left[\left[B_{11}(b), a_{1}\right], a_{2}\right]=0, B_{11}\left(\left[\left[b_{1}, b_{2}\right], b_{3}\right]\right)$ $=0, C_{12}(m b)=C_{12}(m) b+m B_{22}(b)-B_{11}(b) m$.

Proof. Write

$$
L\left(\begin{array}{cc}
a & m \\
& b
\end{array}\right)=\left(\begin{array}{cc}
A_{11}(a)+B_{11}(b)+C_{11}(m) & A_{12}(a)+B_{12}(b)+C_{12}(m) \\
& A_{22}(a)+B_{22}(b)+C_{22}(m)
\end{array}\right)
$$

and we consider $L([[x, y], z])=[[L(x), y], z]+[[x, L(y)], z]+[[x, y], L(z)]$ entrywise.

Take $x=I_{\mathcal{A}}, y=m$ and $z=I_{\mathcal{A}}$, we have

$$
\begin{aligned}
& \left(\begin{array}{cc}
-C_{11}(m) & -C_{12}(m) \\
-C_{22}(m)
\end{array}\right) \\
= & L([[x, y], z]) \\
= & {[[L(x), y], z]+[[x, L(y)], z]+[[x, y], L(z)] } \\
= & \left(\begin{array}{cc}
0 & 2\left(m A_{22}\left(I_{\mathcal{A}}\right)-A_{11}\left(I_{\mathcal{A}}\right)\right) m-C_{12}(m) \\
0
\end{array}\right) .
\end{aligned}
$$

Hence, $C_{11}(m)=0, C_{22}(m)=0$ and $m A_{22}\left(I_{\mathcal{A}}\right)=A_{11}\left(I_{\mathcal{A}}\right) m$. Similarly, if we take $x=I_{\mathcal{A}}, y=m$ and $z=I_{\mathcal{B}}$, we obtain $m B_{22}\left(I_{\mathcal{B}}\right)=B_{11}\left(I_{\mathcal{B}}\right) m$ for every $m \in \mathcal{M}$.

Take $x=a_{1}, y=a_{2}$ and $z=a_{3}$, we have

$$
\begin{aligned}
& \left(\begin{array}{cc}
A_{11}\left(\left[\left[a_{1}, a_{2}\right], a_{3}\right]\right) & A_{12}\left(\left[\left[a_{1}, a_{2}\right], a_{3}\right]\right) \\
A_{22}\left(\left[\left[a_{1}, a_{2}\right], a_{3}\right]\right)
\end{array}\right) \\
= & L([[x, y], z]) \\
= & {[[L(x), y], z]+[[x, L(y)], z]+[[x, y], L(z)] } \\
= & \left(\begin{array}{cc}
{\left[\left[A_{11}\left(a_{1}\right), a_{2}\right], a_{3}\right]+\left[\left[a_{1}, A_{11}\left(a_{2}\right)\right], a_{3}\right]} & a_{3} a_{2} A_{12}\left(a_{1}\right)-a_{3} a_{1} A_{12}\left(a_{2}\right) \\
+\left[\left[a_{1}, a_{2}\right], A_{11}\left(a_{3}\right)\right] & +\left[a_{1}, a_{2}\right] A_{12}\left(a_{3}\right) \\
0
\end{array}\right),
\end{aligned}
$$


from which we see that $A_{11}$ is a Lie triple derivation on $\mathcal{A}$ and $A_{22}\left(\left[\left[a_{1}, a_{2}\right], a_{3}\right]\right)$ $=0$. By taking $a_{1}=a_{3}=I_{\mathcal{A}}$ in (4.1), we have $A_{12}(a)=a A_{12}\left(I_{\mathcal{A}}\right)=a n_{0}$, where we set $n_{0}=A_{12}\left(I_{\mathcal{A}}\right)$. Symmetrically, take $x=b_{1}, y=b_{2}$ and $z=b_{3}$, we obtain $B_{22}$ is a Lie triple derivation on $\mathcal{B}$ and $B_{11}\left(\left[\left[b_{1}, b_{2}\right], b_{3}\right]\right)=0$.

Taking $x=a, y=b_{1}$ and $z=b_{2}$ yields $\left[\left[A_{22}(a), b_{1}\right], b_{2}\right]=0$ and $A_{12}(a) b_{1} b_{2}+$ $a B_{12}\left(b_{1}\right) b_{2}=0$, from which by taking $a=I_{\mathcal{A}}$ and $b_{2}=I_{\mathcal{B}}$ we obtain $B_{12}(b)=$ $-n_{0} b$. Similarly, we have $\left[\left[B_{11}(b), a_{1}\right], a_{2}\right]=0$.

Furthermore, consider $x=a_{1}, y=a_{2}$ and $z=b$, we obtain $B_{11}(b) \in[\mathcal{A}, \mathcal{A}]^{\prime}$. Similarly, by considering $x=b_{1}, y=b_{2}$ and $z=a$, we obtain $A_{22}(a) \in[\mathcal{B}, \mathcal{B}]^{\prime}$.

Finally, let $x=a, y=m$ and $z=I_{\mathcal{B}}$, we obtain $C_{12}(a m)=A_{11}(a) m-$ $m A_{22}(a)+a C_{12}(m)$. Symmetrically, let $x=I_{\mathcal{A}}, y=m$ and $z=b$, we obtain $C_{12}(m b)=C_{12}(m) b+m B_{22}(b)-B_{11}(b) m$.

Conversely, suppose $L$ is a linear map on $\mathfrak{A}=\operatorname{Tri}(\mathcal{A}, \mathcal{M}, \mathcal{B})$ of the form

$$
L\left(\begin{array}{cc}
a & m \\
& b
\end{array}\right)=\left(\begin{array}{cc}
A_{11}(a)+B_{11}(b) & a n_{0}-n_{0} b+C_{12}(m) \\
A_{22}(a)+B_{22}(b)
\end{array}\right)
$$

with conditions (i) and (ii) holding, it is elementary though tedious to check chat $L$ satisfies the relation

$$
L(([x, y], z])=[[L(x), y], z]+[[x, L(y)], z]+[[x, y], L(z)]
$$

for all $x, y, z \in \mathfrak{A}$. We leave it to the readers and the proof is complete.

Remark 4.2. Since $\mathcal{M}$ is faithful, the conditions that $A_{22}\left(\left[\left[a_{1}, a_{2}\right], a_{3}\right]\right)=0$ and $B_{11}\left(\left[\left[b_{1}, b_{2}\right], b_{3}\right]\right)=0$ in Theorem 4.1 are actually not necessary. In fact, for any $a_{1}, a_{2}, a_{3} \in \mathcal{A}$ and $m \in \mathcal{M}$, we have

$$
\begin{aligned}
C_{12}\left(a_{1} a_{2} a_{3} m\right)= & A_{11}\left(a_{1}\right) a_{2} a_{3} m-a_{2} a_{3} m A_{22}\left(a_{1}\right)+a_{1} C_{12}\left(a_{2} a_{3} m\right) \\
= & A_{11}\left(a_{1}\right) a_{2} a_{3} m-a_{2} a_{3} m A_{22}\left(a_{1}\right)+a_{1} A_{11}\left(a_{2}\right) a_{3} m \\
& +a_{1} a_{2} A_{11}\left(a_{3}\right) m-a_{1} a_{3} m A_{22}\left(a_{2}\right)-a_{1} a_{2} m A_{22}\left(a_{3}\right) \\
& +a_{1} a_{2} a_{3} C_{12}(m),
\end{aligned}
$$

from which we obtain

$$
\begin{aligned}
& A_{11}\left(\left[\left[a_{1}, a_{2}\right], a_{3}\right]\right) m-m A_{22}\left(\left[\left[a_{1}, a_{2}\right], a_{3}\right]\right)+\left[\left[a_{1}, a_{2}\right], a_{3}\right] C_{12}(m) \\
= & C_{12}\left(\left[\left[a_{1}, a_{2}\right], a_{3}\right] m\right) \\
= & C_{12}\left(\left(a_{1} a_{2} a_{3}-a_{2} a_{1} a_{3}-a_{3} a_{1} a_{2}+a_{3} a_{2} a_{1}\right) m\right) \\
= & {\left[\left[A_{11}\left(a_{1}\right), a_{2}\right], a_{3}\right] m+\left[\left[a_{1}, A_{11}\left(a_{2}\right)\right], a_{3}\right] m+\left[\left[a_{1}, a_{2}\right], A_{11}\left(a_{3}\right)\right] m } \\
& +\left[\left[a_{1}, a_{2}\right], a_{3}\right] C_{12}(m),
\end{aligned}
$$

whence $A_{22}\left(\left[\left[a_{1}, a_{2}\right], a_{3}\right]\right)=0$ since $\mathcal{M}$ is faithful. Similarly, we have

$$
B_{11}\left(\left[\left[b_{1}, b_{2}\right], b_{3}\right]\right)=0 .
$$

Theorem 4.3. Given a triangular algebra $\mathfrak{A}=\operatorname{Tri}(\mathcal{A}, \mathcal{M}, \mathcal{B})$ with faithful $\mathcal{M}$. $A$ Lie triple derivation $L$ on $\mathfrak{A}$ is proper if and only if $\pi_{\mathcal{A}} L(\mathcal{B}) \subseteq \pi_{\mathcal{A}}(Z(\mathfrak{A})$ ) 
and $\pi_{\mathcal{B}} L(\mathcal{A}) \subseteq \pi_{\mathcal{B}}(Z(\mathfrak{A}))$, where $\mathcal{A}$ and $\mathcal{B}$ are identified as subalgebras of $\mathfrak{A}$. That is to say that a Lie triple derivation $L$ on $\mathfrak{A}$ given by

$$
L\left(\begin{array}{cc}
a & m \\
& b
\end{array}\right)=\left(\begin{array}{cc}
A_{11}(a)+B_{11}(b) & a n_{0}-n_{0} b+C_{12}(m) \\
A_{22}(a)+B_{22}(b)
\end{array}\right)
$$

is proper if and only if $B_{11}(\mathcal{B}) \subseteq \pi_{\mathcal{A}}(Z(\mathfrak{A}))$ and $A_{22}(\mathcal{A}) \subseteq \pi_{\mathcal{B}}(Z(\mathfrak{A}))$.

Proof. Suppose that $L$ is proper and is written as $\delta+h$, where $\delta$ is a derivation and $h(\mathfrak{A}) \subseteq Z(\mathfrak{A})$. By [5, Theorem 2.2.1],

$$
\delta\left(\begin{array}{cc}
a & m \\
& b
\end{array}\right)=\left(\begin{array}{cc}
P(a) & a n-n b+F(m) \\
Q(b)
\end{array}\right)
$$

with $F(a m)=P(a) m+a F(m)$ and $F(m b)=m Q(b)+F(m) b$, whereas by the structure of $Z(\mathfrak{A})$,

$$
h\left(\begin{array}{cc}
a & m \\
& b
\end{array}\right)=\left(\begin{array}{cc}
G_{1}(a)+G_{2}(m)+G_{3}(b) & 0 \\
& H_{1}(a)+H_{2}(m)+H_{3}(b)
\end{array}\right) .
$$

Since $L=\delta+h$, we have $n=n_{0}, F=C_{12}, G_{2}=0, H_{2}=0, A_{11}=P+G_{1}$, $A_{22}=H_{1}, B_{11}=G_{3}$ and $B_{22}=Q+H_{3}$. Hence

$$
\begin{aligned}
& B_{11}(b)=G_{3}(b)=\pi_{\mathcal{A}}(h(b)) \subseteq \pi_{\mathcal{A}}(Z(\mathfrak{A})), \\
& A_{22}(a)=H_{1}(a)=\pi_{\mathcal{B}}(h(a)) \subseteq \pi_{\mathcal{B}}(Z(\mathfrak{A})) .
\end{aligned}
$$

Conversely, suppose $B_{11}(\mathcal{B}) \subseteq \pi_{\mathcal{A}}(Z(\mathfrak{A}))$ and $A_{22}(\mathcal{A}) \subseteq \pi_{\mathcal{B}}(Z(\mathfrak{A}))$. Define

$$
\begin{aligned}
& \delta\left(\begin{array}{cc}
a & m \\
& b
\end{array}\right)=\left(\begin{array}{cc}
A_{11}(a)-\tau^{-1}\left(A_{22}(a)\right) & a n_{0}-n_{0} b+C_{12}(m) \\
B_{22}(b)-\tau B_{11}(b)
\end{array}\right), \\
& h\left(\begin{array}{cc}
a & m \\
& b
\end{array}\right)=\left(\begin{array}{cc}
B_{11}(b)+\tau^{-1}\left(A_{22}(a)\right) & 0 \\
& A_{22}(a)+\tau B_{11}(b)
\end{array}\right) .
\end{aligned}
$$

It is easy to verify that $h(\mathfrak{A}) \subseteq Z(\mathfrak{A})$ and $h([[x, y], z])=0$ for all $x, y, z \in \mathfrak{A}$. Since

$$
\begin{aligned}
C_{12}(a m) & =A_{11}(a) m-m A_{22}(a)+a C_{12}(m) \\
& =\left(A_{11}(a)-\tau^{-1}\left(A_{22}(a)\right)\right) m+a C_{12}(m) \quad \text { and } \\
C_{12}(m b) & =C_{12}(m) b+m B_{22}(b)-B_{11}(b) m \\
& =C_{12}(m) b+m\left(B_{22}(b)-\tau B_{11}(b)\right),
\end{aligned}
$$

$\delta$ is a derivation by [5, Corollary 2.2.2].

Remark 4.4. In Theorem 4.3, the condition that $\mathcal{M}$ is faithful is not necessary for the necessity part of the proof, where in fact, we didn't use this condition. That is, without the condition $\mathcal{M}$ is faithful, if a Lie triple derivation $L$ on $\mathfrak{A}$ given by

$$
L\left(\begin{array}{cc}
a & m \\
& b
\end{array}\right)=\left(\begin{array}{cc}
A_{11}(a)+B_{11}(b) & a n_{0}-n_{0} b+C_{12}(m) \\
A_{22}(a)+B_{22}(b)
\end{array}\right)
$$

is proper, then we have $B_{11}(\mathcal{B}) \subseteq \pi_{\mathcal{A}}(Z(\mathfrak{A}))$ and $A_{22}(\mathcal{A}) \subseteq \pi_{\mathcal{B}}(Z(\mathfrak{A}))$. 
Clearly, every Lie derivation is a Lie triple derivation. In [6, Example 8], Cheung gave an example of a triangular algebra which has an improper Lie derivation. In the following, we give an example of a triangular algebra which has an improper Lie triple derivation that is not a Lie derivation.

Example 4.5. Let

$$
\mathcal{A}=\mathcal{B}=\mathcal{M}=\left\{\left(\begin{array}{ccc}
0 & t_{1} & p \\
& 0 & t_{2} \\
& & 0
\end{array}\right): t_{1}, t_{2}, p \in \mathbb{C}\right\} .
$$

Then the map

$$
L:\left(\begin{array}{cccccc}
0 & t_{1} & p & 0 & a & b \\
& 0 & t_{2} & & 0 & c \\
& & 0 & & & 0 \\
& & & 0 & s_{1} & q \\
& & & & 0 & s_{2} \\
& & & & & 0
\end{array}\right) \longrightarrow\left(\begin{array}{cccccc}
0 & s_{1} & q & 0 & 0 & 0 \\
& 0 & s_{2} & & 0 & 0 \\
& & 0 & & & 0 \\
& & & 0 & t_{1} & p \\
& & & & 0 & t_{2} \\
& & & & & 0
\end{array}\right)
$$

on $\mathfrak{A}=\operatorname{Tri}(\mathcal{A}, \mathcal{M}, \mathcal{B})$ is an improper Lie triple derivation that is not a Lie derivation.

Proof. For any $x, y, z \in \mathfrak{A}, L$ is a Lie triple derivation since $L([[x, y], z])=0=$ $[[L(x), y], z]+[[x, L(y)], z]+[[x, y], L(z)]$. If we take

$$
x=\left(\begin{array}{cccccc}
0 & 2 & 0 & 0 & 0 & 0 \\
& 0 & 1 & & 0 & 0 \\
& & 0 & & & 0 \\
& & & 0 & 1 & 0 \\
& & & & 0 & 1 \\
& & & & & 0
\end{array}\right) \text { and } y=\left(\begin{array}{cccccc}
0 & 1 & 0 & 0 & 0 & 0 \\
& 0 & 2 & & 0 & 0 \\
& & 0 & & & 0 \\
& & & 0 & 1 & 0 \\
& & & & 0 & 1 \\
& & & & & 0
\end{array}\right) \text {, }
$$

then

$$
L([x, y])=\left(\begin{array}{cccccc}
0 & 0 & 0 & 0 & 0 & 0 \\
& 0 & 0 & & 0 & 0 \\
& & 0 & & & 0 \\
& & & 0 & 0 & 3 \\
& & & & 0 & 0 \\
& & & & & 0
\end{array}\right)
$$

while

$$
[L(x), y]+[x, L(y)]=\left(\begin{array}{cccccc}
0 & 0 & 2 & 0 & 0 & 0 \\
& 0 & 0 & & 0 & 0 \\
& & 0 & & & 0 \\
& & & 0 & 0 & 2 \\
& & & & 0 & 0 \\
& & & & & 0
\end{array}\right) \neq L([x, y])
$$


that is, $L$ is not a Lie derivation. Note that

$$
\pi_{\mathcal{A}}(Z(\mathfrak{A}))=\pi_{\mathcal{B}}(Z(\mathfrak{A}))=\left\{\left(\begin{array}{ccc}
0 & 0 & p \\
& 0 & 0 \\
& & 0
\end{array}\right): p \in \mathbb{C}\right\} .
$$

Since

$$
B_{11}\left(\begin{array}{ccc}
0 & s_{1} & q \\
& 0 & s_{2} \\
& & 0
\end{array}\right)=\left(\begin{array}{ccc}
0 & s_{1} & q \\
& 0 & s_{2} \\
& & 0
\end{array}\right) \notin \pi_{\mathcal{A}}(Z(\mathfrak{A})),
$$

we have $B_{11}(\mathcal{B}) \nsubseteq \pi_{\mathcal{A}}(Z(\mathfrak{A}))$, and thus $L$ is improper by Theorem 4.3 and Remark 4.4.

For an algebra $\mathcal{A}$, we denote $W(\mathcal{A})$ the subalgebra of $\mathcal{A}$ generated by commutators and idempotents.

Theorem 4.6. Every Lie triple derivation of $\mathfrak{A}=\operatorname{Tri}(\mathcal{A}, \mathcal{M}, \mathcal{B})$ with faithful $\mathcal{M}$ is proper if

(1) $W(\mathcal{A})^{\prime} \cap \mathcal{A} \subseteq \pi_{\mathcal{A}}(Z(\mathfrak{A}))$,

(2) $W(\mathcal{B})^{\prime} \cap \mathcal{B} \subseteq \pi_{\mathcal{B}}(Z(\mathfrak{A}))$.

Proof. By Proposition 4.1 and Theorem 4.3, we only need to show that for any idempotent $e \in \mathcal{A}$ and $b \in \mathcal{B}, B_{11}(b) e=e B_{11}(b)$.

For any $a_{1}, a_{2} \in \mathcal{A}$ and $b \in B$, Proposition 4.1 gives $\left[\left[B_{11}(b), a_{1}\right], a_{2}\right]=0$, from which we have

$$
a_{2} B_{11}(b) a_{1}+a_{1} B_{11}(b) a_{2}=B_{11}(b) a_{1} a_{2}+a_{2} a_{1} B_{11}(b) .
$$

Taking $a_{1}=a_{2}=e$ in (4.2) gives $B_{11}(b) e=e B_{11}(b)$. The proof for the other case is similar.

Two corollaries describing Lie triple derivations of (block) upper triangular matrix algebras and (continuous) nest algebras follow directly from our main theorem.

Corollary 4.7. Let $\mathcal{R}$ be a commutative ring with identity. Every Lie triple derivation on $B_{n}^{\bar{k}}(\mathcal{R})\left(\neq M_{n}(\mathcal{R})\right)$ is proper. In particular, every Lie triple derivation on $T_{n}(\mathcal{R})$ is proper.

Proof. It is trivial when $n=1$.

Suppose $n \geq 2$. Let $e_{i j}$ be the $n \times n$ matrix with its $(i, j)$-entry equals 1 and other entries equal 0 . Then $e_{i j}$ is an idempotent if $i=j$ and $e_{i j}=\left[e_{i i}, e_{i j}\right]$ is a commutator if $i \neq j$. Thus if we regard the block upper triangular matrix algebra as a triangular algebra of the form

$$
B_{n}^{\bar{k}}(\mathcal{R})=\left(\begin{array}{cc}
B_{l}^{\overline{k_{1}}}(\mathcal{R}) & M_{l \times(n-l)}(\mathcal{R}) \\
& B_{n-l}^{k_{2}}(\mathcal{R})
\end{array}\right)=\left(\begin{array}{cc}
\mathcal{A} & \mathcal{M} \\
& \mathcal{B}
\end{array}\right),
$$

where $l \in\left\{k_{1}, k_{1}+k_{2}, \ldots, k_{1}+k_{2}+\cdots+k_{m-1}\right\}$ and $\overline{k_{1}} \in \mathbb{N}^{l}, \overline{k_{2}} \in \mathbb{N}^{m-l}$, then $W(\mathcal{A})=\mathcal{A}$ and $W(\mathcal{B})=\mathcal{B}$. Since $Z\left(B_{n}^{\bar{k}}\right)=\mathcal{R} 1, Z(\mathcal{A})=\mathcal{R} 1_{\mathcal{A}}$ and $Z(\mathcal{B})=\mathcal{R} 1_{\mathcal{B}}$, our conclusion follows immediately from Theorem 4.6. 
Let $\mathcal{N}$ be a nest on a Hilbert space $H$ and $\operatorname{alg} \mathcal{N}$ be the associated algebra. If $\mathcal{N}$ is trivial, then $\operatorname{alg} \mathcal{N}$ is $B(H)$. If $\mathcal{N}$ is nontrivial, take a nontrivial projection $P \in \mathcal{N}$. Let $\mathcal{A}=P \operatorname{alg} \mathcal{N} P, \mathcal{M}=P \operatorname{alg} \mathcal{N}(I-P)$ and $\mathcal{B}=(I-P) \operatorname{alg} \mathcal{N}(I-P)$. Then $\mathcal{M}$ is a faithful $(\mathcal{A}, \mathcal{B})$-bimodule, and $\operatorname{alg} \mathcal{N}=\operatorname{Tri}(\mathcal{A}, \mathcal{M}, \mathcal{B})$ is an upper triangular algebra. A nest $\mathcal{N}$ is said to be continuous if for every $N \in \mathcal{N}$, $\inf \{M \in \mathcal{N}: N \varsubsetneqq M\}=N$. For instance, let $H_{t}=\left\{f \in L^{2}[0,1]: f(x)=\right.$ 0 for $0 \leq x \leq t\}$, then $\left\{H_{t}: 0 \leq t \leq 1\right\}$ is a continuous nest. By [8, Proposition $2.6]$, every element of the nest algebra of a continuous nest is a sum of two commutators. Hence the following corollary is obvious.

Corollary 4.8 ([15]). Let $\mathcal{N}$ be a continuous nest on a Hilbert space $H$ and alg $\mathcal{N}$ be the associated algebra. Then every Lie triple derivation on alg $\mathcal{N}$ is proper.

Acknowledgement. The authors are thankful to the referee for careful reading of the paper and valuable suggestions. This work is supported by NSF of China.

\section{References}

[1] D. Benkovič, Biderivations of triangular algebras, Linear Algebra Appl. 431 (2009), no. 9, 1587-1602.

[2] D. Benkovič and D. Eremita, Commuting traces and commutativity preserving maps on triangular algebras, J. Algebra 280 (2004), no. 2, 797-824.

[3] M. Brešar, Commuting traces of biadditive mappings, commutativity-preserving mappings and Lie mappings, Trans. Amer. Math. Soc. 335 (1993), no. 2, 525-546.

[4] W. S. Cheung, Commuting maps of triangular algebras, J. London Math. Soc. 63 (2001), no. $1,117-127$

[5] _ Mappings on triangular algebras, Ph. D Dissertation, University of Victoria, 2000.

[6] L Lie derivations of triangular algebras, Linear Multilinear Algebra 51 (2003), no. 3, 299-310.

[7] F. Y. Lu, Lie triple derivations on nest algebras, Math. Nachr. 280 (2007), no. 8, 882887.

[8] L. W. Marcoux and A. R. Sourour, Lie isomorphisms of nest algebras, J. Funct. Anal. 164 (1999), no. 1, 163-180.

[9] C. R. Miers, Lie triple derivations of von Neumann algebras, Proc. Amer. Math. Soc. 71 (1978), no. 1, 57-61.

[10] A. Nakajima, On generalized higher derivations, Turkish J. Math. 24 (2000), no. 3, $295-311$.

[11] X. F. Qi and J. C. Hou, Lie higher derivations on Nest Algebras, Commun. Math. Res. 26 (2010), no. 2, 131-143

[12] H. T. Wang and Q. G. Li, Lie triple derivation of the Lie algebra of strictly upper triangular matrix over a commutative ring, Linear Algebra Appl. 430 (2009), no. 1, 66-77.

[13] T. L. Wong, Jordan isomorphisms of triangular rings, Proc. Amer. Math. Soc. 133 (2005), no. 11, 3381-3388.

[14] Z. K. Xiao and F. Wei, Jordan higher derivations on triangular algebras, Linear Algebra Appl. 432 (2010), no. 10, 2615-2622.

[15] J. H. Zhang, B. W. Wu, and H. X. Cao, Lie triple derivations of nest algebras, Linear Algebra Appl. 416 (2006), no. 2-3, 559-567. 
[16] J. H. Zhang and W. Y. Yu, Jordan derivations of triangular algebras, Linear Algebra Appl. 419 (2006), no. 1, 251-255.

JIANKUI LI

Department of Mathematics

East China University of Science and Technology

Shanghai 200237, P. R. China

E-mail address: jiankuili@yahoo.com

QIHUA SHEN

Department of Mathematics

East China University of Science and Technology

Shanghai 200237, P. R. China

E-mail address: qihuashen@yahoo.com.cn 